\title{
Sicurezza alimentare e processi di integrazione della regolazione pubblica e privata
}

\author{
Giuseppina Carrà, luri Peri \\ Dipartimento Gestione dei Sistemi Agroalimentari e Ambientali, Università di Catania, Italy
}

\begin{abstract}
A new paradigm of regulation and management of food safety and quality has been gradually gaining ground. It is based both on the evolution of public standards and the increasing involvement of the private sector, which promotes forms of self-regulation, by adopting standards whose requirements are often higher than public ones. That reflects the needs and wants of consumers to obtain better guarantees on food safety and quality requirements and, consequently, the crucial role that these requirements have in supporting the enterprise strategy in the present day competitive market. The emergence of private standards, operating in various modes of interaction side-by-side with the public standards, has an important impact on the governance of agri-food supply chains. Looking at the literature from national and international perspective, the paper addresses, after an introduction on the role of standards in response to market failures, the incentives for firms to improve food safety and quality by adopting private standards. Then, it examines the integration processes of public and private standards and the related problems of governance of agri-food supply chains, operating increasingly at global dimension. These problems are associated to dominant role of private governance structures in agri-food markets, whose impact is the subject of an ongoing debate. The paper concludes by suggesting potential evolving of some key elements.
\end{abstract}

Correspondence: Iuri Peri, Dipartimento Gestione dei Sistemi Agroalimentari e Ambientali, Università di Catania, via Santa Sofia 100, 95123 Catania, Italy.

Tel. +39.095 .7580313 - Fax: +39.095 .7580345 .

E-mail: peri@unict.it

Key words: agri-food, governance, quality, safety, supply chain.

Parole chiave: agro-alimentare, catene di offerta, governance, qualità, sicurezza.

Received for publication: 31 May 2011.

Accepted for publication: 1 July 2011.

Società rappresentata nell'ambito AISSA: Società Italiana di Economia Agraria - SIDEA

(C) Copyright G. Carrà and I. Peri 2011

Under no circumstances figures can be used without prior written consent of the copyright owner.

Licensee PAGEPress, Italy

Italian Journal of Agronomy 2011; 6(s2):e9

doi:10.4081/ija.2011.6.s2.e9

This work is licensed under a Creative Commons Attribution NonCommercial 3.0 License (CC BY-NC 3.0).

\section{Riassunto}

Un nuovo paradigma di regolazione e gestione della sicurezza e qualità alimentare si è andato via via affermando. Esso vede, in parallelo all'evoluzione delle norme pubbliche, il crescente coinvolgimento del settore privato che promuove forme di auto-regolazione, con l'adozione di standard i cui requisiti sono spesso più stringenti di quelli pubblici. Tutto ciò riflette le esigenze e le attese da parte del consumatore di conseguire maggiori garanzie in merito ai requisiti di qualità e sicurezza degli alimenti e, conseguentemente, il ruolo che tali requisiti assumono come fattore competitivo per l'impresa. L'affermarsi degli standard privati, che operano in un complesso intreccio accanto a quelli pubblici, ha effetti importanti sulla governance delle catene di offerta agroalimentare. Sulla base dell'ampia letteratura sviluppatasi su questo tema, il paper esamina, dopo una parte introduttiva sul ruolo degli standard come risposta ai fallimenti del mercato, gli incentivi che spingono le imprese a migliorare il livello di sicurezza e qualità degli alimenti. L'analisi prosegue mettendo in luce i processi d'integrazione della regolazione pubblica e privata e i connessi problemi di governance delle catene di offerta agroalimentari, le quali operano in una dimensione sempre più globale. Tali problemi sembrano evidenziarsi in rapporto al ruolo predominante che tendono a occupare le strutture private di governance, il cui impatto è oggetto di un acceso dibattito. Il paper si conclude con l'indicazione di alcuni potenziali elementi di evoluzione.

\section{Introduczione}

La sicurezza alimentare è un aspetto imprescindibile della qualità degli alimenti, nella misura in cui precede tutte le altre considerazioni, essendo concettualmente integrata nella qualità del prodotto. Essa può inoltre corrispondere a delle esigenze che vanno ben di là dell'assenza di fattori di rischio che possono causare danni alla salute a seguito del consumo di un certo alimento, collegandosi a considerazioni che hanno a che fare con la qualità della vita (Zamagni, 2006). In questa visione più ampia, la sicurezza alimentare è assimilata a un modello di produzione e consumo sostenibile e rispondente a requisiti di ordine etico, ${ }^{1}$ cui le società avanzate sono sempre più sensibili (de Stefano, 2009).

${ }^{I}$ Coff et al. (2008) individuano i problemi etici relativi alla catena di offerta alimentare, distinguendoli in problemi di natura sostanziale e procedurale. La prima categoria comprende le preoccupazioni per il benessere degli animali; la salute umana (in relazione alla sicurezza e igiene alimentare, alle malattie degli animali, alle condizioni di lavoro, ecc); i metodi di produzione e di trasformazione e il loro impatto sull'ambiente e il paesaggio, sul benessere degli animali; le ragioni di scambio (commercio equo, ecc); le condizioni di lavoro; le caratteristiche intrinseche di qualità dei prodotti (gusto, composizione, ecc), luogo di origine. La seconda categoria di problemi fa riferimento alle relazioni che regolano gli scambi tra gli operatori lungo la catena di offerta, quali fiducia, partecipazione e trasparenza. La lista dei potenziali problemi etici è destinata ad evolversi nel tempo in ragione dell'emergere di nuove e diverse esigenze e valori in seno alla società. 
Poiché gli attributi di qualità e sicurezza alimentare sono difficili da valutare, si sostiene che a tale riguardo i mercati spesso falliscono. In particolare, alcuni rischi associati con gli alimenti non possono essere valutati né prima né dopo il consumo (attributi credence degli alimenti), generando fenomeni d'imperfetta informazione (incompletezza e asimmetria informativa). Considerata nel suo insieme, la letteratura ha identificato un set di distinti problemi connessi ai fenomeni di imperfetta informazione: comportamento sleale, selezione avversa, elevati costi di transazione. I primi due problemi derivano dal fatto che i prodotti con attributi di sicurezza e qualità più elevati sono più costosi da produrre rispetto a quelli meno sicuri e che tali attributi non sono osservabili. In particolare, il comportamento sleale riguarda forme di opportunismo che può portare i venditori a perseguire i propri interessi a spese degli acquirenti, confidando nella impossibilità, per questi ultimi, di verificare la presenza dei requisiti attesi. La selezione avversa si riferisce alla tendenza secondo cui i prodotti con requisiti di sicurezza e qualità migliori sono soppiantati dai prodotti a più elevato rischio e di qualità peggiore (Akerlof, 1970). Allo stesso tempo, i costi di transazione associati con la valutazione di questi attributi tendono a essere elevati. L'ammontare d'informazione disponibile, dunque, è cruciale per il mercato.

Tenuto conto delle esternalità e delle asimmetrie d'informazione, i mercati non riescono da soli a garantire un grado di qualità e sicurezza auspicabile per la collettività, costituendo ciò la principale giustificazione dell'intervento pubblico a carattere cogente in questa materia. Per lo più, gli standard di sicurezza e qualità definiti dall'autorità pubblica nelle transazioni riguardanti i prodotti agroalimentari assumono caratteristiche di bene pubblico (non escludibilità, non rivalità) e costituiscono requisiti essenziali di ogni sistema agroalimentare moderno. L'autorità pubblica può ugualmente introdurre norme che disciplinano standard di qualità volontari la cui osservanza è obbligatoria per i privati che intendono conformarsi. Anche l'iniziativa privata interviene determinando un'ampia gamma di standard volontari, ma che in determinate condizioni possono divenire obbligatori, di fatto, da un punto di vista commerciale (Henson e Humphrey, 2008). È stato evidenziato come l'evoluzione degli standard alimentari pubblici e privati, che operano con modalità sempre più complesse d'interazione a livello nazionale e internazionale, sia frutto di alcune dinamiche manifestatesi prepotentemente negli ultimi decenni. Queste comprendono la globalizzazione del commercio; i progressi della tecnologia dell'informazione; le riforme dei sistemi di regolazione della sicurezza alimentare come risposta ad una serie di crisi e scandali alimentari; il cambiamento delle preferenze dei consumatori e il maggiore interesse sia tra i consumatori e sia tra le imprese verso i processi di produzione alimentare e, più in generale, le mutate concezioni di sicurezza e qualità alimentare; la concentrazione delle industrie alimentari e delle imprese distributive; le strategie competitive delle imprese basate sulla differenziazione dei prodotti; la crescente complessità e globalizzazione delle catene di offerta alimentare che coinvolgono più sistemi di regolazione, paesi e operatori (Liu, 2009; Henson and Humphrey, 2009; FA0, 2010).

\section{Standard privati e incentivi per le imprese}

Gli standard privati hanno registrato in anni recenti un considerevole incremento (Henson e Reardon, 2005; Giraud-Héraud et al. 2008). Essi si riferiscono a una vasta gamma di regole e requisiti prevalentemente sviluppati dalla grande distribuzione, ma anche dalle catene di ristorazione, oltre che da parte dei produttori e di altri operatori lungo la catena di offerta, nonché di organizzazioni che rappresentano gli interessi dei consumatori e della società civile ${ }^{2}$ (FA0, 2010). La maggior parte degli standard privati affronta aspetti attinenti la sicurezza alimentare, con una crescente attenzione verso i processi di produzione, ma possono fare riferimento ad altri attributi della qualità degli alimenti e comprendere aspetti etici ed ambientali. Essi sono sempre più oggetto di controlli attraverso certificazione da parte di enti terzi ${ }^{3}$ (FAO, 2010).

Se il motivo alla base dell'intervento pubblico nella regolazione del settore agroalimentare è quello di contenere gli effetti derivanti dai fallimenti del mercato, per l'iniziativa privata finalizzata alla definizione di standard le motivazioni possono essere diverse.

Il ricorso a standard e a sistemi di regole definite autonomamente dal settore privato trova giustificazione, in primo luogo, nella mitigazione dei rischi di reputazione e/o commerciali associati alla sicurezza dei prodotti alimentari, per cui si presume che una regolazione pubblica sempre più pressante abbia indotto i produttori a conformarsi progressivamente a più elevati standard di qualità e sicurezza (Green e Perito, 2008). Pertanto, le imprese sarebbero spinte ad adottare standard privati anche al fine di conformarsi alle norme pubbliche ed evitare così i costi potenziali derivanti da responsabilità legali, nell'eventualità che si possano verificare delle crisi di sicurezza alimentare.

Alcuni studi hanno dimostrato come gli incentivi a investire nella sicurezza alimentare dipenda dall'entità dei costi che possono derivare dal fornire prodotti non sicuri e dalla loro ripartizione all'interno della catena di offerta. Si tratta di incentivi che spingono verso una qualità che va oltre il rispetto dei requisiti minimi e che va intesa come conformità al mercato di riferimento. I dettaglianti, in particolare, hanno forti incentivi a fissare propri standard per garantire la salubrità e la sicurezza degli alimenti, giacché essi sono gli operatori maggiormente responsabili per la traslazione della domanda dei consumatori (Hammoudi et al., 2009). È stato osservato, inoltre, che la regolazione pubblica sempre più frequentemente si trova a dettare i requisiti in materia di qualità del prodotto finale, lasciando in qualche misura alle singole imprese di scegliere il modo più adeguato per raggiungere questo risultato. Ciò in larga misura è stato realizzato adottando e imponendo ai fornitori di adottare diversi standard privati che sono più rigorosi dei requisiti di legge (Hammoudi et al., 2009). L'iniziativa privata tende ad avere un ruolo sostitutivo dell'intervento pubblico laddove questo risulti carente (Reardon et al., 2001), oppure di complemento, quando si verifichi la necessità, proprio per la realizzazione compiuta degli standard previsti a livello pubblico, di sviluppare standard privati armonizzati con quelli pubblici preesistenti (Henson e Reardon, 2005). Una regolazione pubblica troppo esigente, tuttavia, avrebbe come effetto quello di scoraggiare gli investimenti in direzione di un miglioramento della qualità alimentare; cioè, il settore privato potrebbe perdere l'interesse strategico a sviluppare uno standard più stringente rispetto a quello definito dalla legislazione. Viceversa, requisiti imposti dall'autorità pubblica di grado moderato potrebbero favorire lo sviluppo di iniziative private mirate ad incrementare il livello di qualità degli alimenti (Giraud-Héraud, 2006; Malorgio e Grazia, 2009).Per questa ragione può dirsi che gran parte degli standard privati è collegata, a sua volta, al livello e alla natura dei requisiti normativi, anche perché le imprese seguono una strategia di differenziazione a partire da uno standard minimo di qualità richiesto dalle norme obbligatorie, per cui il livello degli standard pubblici influenza quello degli standard privati al fine di mantenere un certo grado di differenziazione del prodotto e, quindi, il suo posizionamento competitivo sul mercato (Codron et al., 2005).

${ }^{2}$ Per la classificazione degli standard privati si rinvia a Henson e Humphrey (2009) e WTO (2007), che identificano tre principali tipologie: standard collettivi internazionali (es., GlobalGAP), standard collettivi nazionali (es., British Retail Consortium Global Standard Food) e standard di singole imprese (es., Carrefour Filière Qualité), a fronte di una lista molto diversificata di oltre 400 sistemi operanti.

${ }^{3}$ Si individuano due grandi categorie di sistemi di garanzia e certificazione della qualità. quelli indicati con l'acronimo OSA (Ouality Assurance System) e i sistemi di gestione della qualità, indicati con l'acronimo QMS (Quality Management System). I primi hanno lo scopo di segmentare il mercato proteggendo un prodotto che presenta caratteristiche specifiche, distintive rispetto a quelle di prodotti analoghi (ad es. DOP, IGP, STG e Marchio Biologico). Si tratta di schemi che si avvalgono di simboli o marchi per segnalare ai consumatori le qualità del processo utilizzato e del prodotto. I secondi includono sistemi di certificazione generali come quelli che si basano sugli standard definiti da ISO o dal settore privato (es. BRC, IFS, GlobalGAP) che hanno una segnalazione interaziendale e solitamente non vengono comunicati al consumatore. 
L'operare degli incentivi di tipo negativo, cioè miranti a evitare eventi avversi per le imprese, non basta da solo a giustificare lo sviluppo degli standard volontari che vanno oltre i requisiti legali. Lo sviluppo di standard privati è spiegato anche dall'esistenza di vantaggi competitivi attesi e connessi allo stimolo esercitato dai consumatori, in ragione della fiducia che essi ripongono nei segnali estrinseci della sicurezza e della qualità, sopratutto nel caso degli attributi credence. ${ }^{4}$ Gli standard privati fanno riferimento, quindi, ad un'ampia gamma di attributi degli alimenti che vanno oltre i requisiti di sicurezza e qualità dei prodotti, interessando aspetti ambientali e sociali (Henson and Reardon, 2005). Pertanto, l'adozione di standard volontari di sicurezza e qualità, rispondendo alle esigenze dei consumatori, consentirebbe alle imprese di cogliere l'opportunità di differenziare la propria produzione. I vantaggi derivanti dalla scelta strategica di comunicare i requisiti qualitativi sono poi collegati al posizionamento dei prodotti differenziati nei segmenti a più alto valore dei mercati agricoli e alimentari. Infine, l'adozione di standard privati può rappresentare uno strumento di gestione strategica per conseguire vantaggi competitivi, per la spinta esercitata a realizzare forme di coordinamento verticale e orizzontale. I vantaggi attesi dall'adozione di standard privati vengono ricondotti agli effetti positivi generati dai sistemi di gestione della qualità in termini di miglioramento dell'efficienza del processo produttivo (ad es., incremento della produttività) (Swinnen e Maertens, 2007), di esternalità prodotte (ad es., riduzione dei costi di transazione, esternalità di rete) (Henson e Caswell, 1999; Smith, 2009).

Le imprese della moderna distribuzione al dettaglio guidano iniziative di normalizzazione e modalità di coordinamento delle catene di offerta che si sono dimostrate efficaci (Ménard e Valceschini, 2005; Pulina, 2010). Oltre ai sistemi promossi dalla moderna distribuzione al dettaglio, le iniziative sostenute dai produttori e da altri operatori lungo la catena di offerta evidenziano un interesse crescente verso forme organizzate degli scambi di tipo ibrido, tra mercato e gerarchia. ${ }^{5}$ Queste coprono un'ampia gamma di strutture di relazioni, dalle alleanze strategiche tra imprese, agli accordi di franchising, ai marchi collettivi, alle associazioni di produttori, ecc., che prevedono relazioni più stabili, a carattere complementare, competitivo, reciproco, negoziale (Ménard, 2004; Rama, 2010).

In definitiva, gli standard privati si sono evoluti in risposta agli sviluppi nella regolazione e alla crescente attenzione e preoccupazione dei consumatori, come strumento di posizionamento competitivo sul mercato per prodotti agroalimentari di elevata qualità (World Bank, 2005), nonché come adattamento efficiente delle imprese alle specifiche caratteristiche dei diversi segmenti del mercato. Il loro successo poggia sugli incentivi basati sul mercato che spingono le imprese a investire in una migliore qualità alimentare e in maggiori controlli della catena di offerta.

\section{Regolazione pubblico-privata e sistemi di governance}

Gli standard di sicurezza e qualità degli alimenti costituiscono principi funzionali essenziali della governance dei mercati agroalimentari che influiscono sul modo di produrre, trasformare e vendere gli alimenti. In una prospettiva dinamica, che prende in considerazione i cambiamenti strutturali della catena di offerta e i processi di regolazione del settore pubblico e di quello privato, si pone anche il filone di studi incentrato sui sistemi di governance. La riflessione a questo riguardo coinvolge l'evoluzione dei modelli normativi, con il passaggio da azioni di comando e controllo a forme indirette di direzione da parte dell'autorità pubblica.

La regolazione pubblica della catena di offerta agro-alimentare, di fatto, guida il settore privato verso forme di auto-regolazione che ampliano e approfondiscono la portata delle norme. Questa tendenza è evidente in ambito europeo, dove si è assistito al passaggio da standard obbligatori dal punto di vista giuridico, principalmente di competenza dell'autorità pubblica, a forme di regolazione volontaria attivamente promosse dal settore privato. Un tale sviluppo delle politiche di regolazione ha finito col trasferire al settore privato la maggior parte dei costi economici oltre che la responsabilità per la sicurezza degli alimenti, seppure, com'è stato osservato da Smith (2009), sui mercati delle commodities gli standard di sicurezza abbiano caratteristiche di beni pubblici e, pertanto, i relativi sistemi di regolazione resteranno anche in futuro prevalentemente appannaggio delle autorità pubbliche.

Più generalmente, questa evoluzione riflette la crescente importanza dei meccanismi di soft governance nei sistemi economici nazionali e internazionali e, in particolare, nei modelli di governance europea. Ad essi si ispirano, ad esempio, i processi d'innovazione dei sistemi di regolazione attraverso l'approccio di co-regolazione (Garcia Martinez et al., 2007), che permette a tutte le parti, pubbliche e private, coinvolte in un determinato mercato, di cooperare nella definizione e applicazione degli standard di riferimento (Green e Perito, 2008).

I molteplici spazi di regolazione e gestione della qualità e della sicurezza alimentare occupati dal settore privato - spazi tradizionalmente pensati come esclusivi delle autorità pubbliche - hanno spostato l'attenzione degli studiosi dai regimi di regolazione pubblici a quelli privati. La svolta ha segnato anche un cambiamento del focus dagli aspetti più propriamente connessi all'assetto degli standard ai sistemi di governance. A livello privato, questi fanno capo ad istituzioni la cui autorità nel mercato globale non deriva dai governi, ma emana dalla catena di offerta (Bernstein e Cashore, 2007).

Il passaggio di competenze verso il settore privato ha creato una governance più complessa, per cui la riflessione riguarda il modo in cui formulare politiche negoziate che perseguono vantaggi sia sociali sia privati (Garcia Martinez et al., 2007). Nonostante la co-regolazione sia un processo ancora poco sviluppato (Smith, 2009), il tema del coordinamento tra regolazione pubblica e privata richiama una crescente attenzione per i suoi riflessi sulla governance del sistema agroalimentare. Il dibattito scientifico ne segnala l'importanza al fine di migliorare l'efficacia del sistema complessivo a costi inferiori e rendere disponibili nei mercati nazionali e globali alimenti più sicuri e di migliore qualità (Henson e Humphrey, 2009).

È evidente come questa prospettiva d'integrazione dipenda dai diversi contesti di regolazione: ad esempio, quello europeo sembra offrire buone opportunità in questa direzione, ma non va confinata ai soli aspetti normativi (formulazione degli standard), piuttosto è il modo di operare degli strumenti che, al di là di una rappresentazione generalizzata di finalità comuni, può determinare effetti diversi quanto a coerenza, visibilità e responsabilità dei suoi risultati. ${ }^{6}$

Il dibattito riguardo agli effetti derivanti dal modo di operare degli strumenti di governance privati resta tuttora aperto. Esso ha investito diverse sedi internazionali (UNCTAD, 2007; OECD, 2007; WTO, 2007), oltre ad aver stimolato una vasta letteratura, che si concentra principalmente su alcuni problemi che qui di seguito si richiamano.

${ }^{4} \mathrm{Di}$ fatto, $\mathrm{i}$ consumatori tendono a reagire alla percezione del rischio piuttosto che al rischio oggettivo di acquistare alimenti poco sicuri (Grunert, 2005).

${ }^{5}$ Nella prospettiva della teoria dei costi di transazione (TCE), le forme organizzative ibride si riferscono a strutture di governance delle transazioni che si collocano in un continuum rappresentato da diversi gradi di coordinamento, $i$ cui estremi sono rappresentati dai mercati spot, da un lato, e dall'integrazione verticale, dall'altro (Williamson, 1996).

${ }^{6}$ Il governo attraverso gli strumenti designa una forma di governo debole che opera a distanza e che si fonda sul principio del far fare e anche del lasciar fare più che del fare: un governo che orienta, incentiva, vincola, le azioni e interazioni di una pluralità di attori dentro processi aperti e incerti, investendo dunque su effetti organizzativi prima che su obiettivi di merito (Lascoumes e Le Galès, 2009). 


\section{Standard volontari ma obbligatori di fatto}

Benché gli standard privati siano nominalmente volontari, possono risultare per i fornitori che fanno parte di un certo mercato, obbligatori di fatto (Henson e Northen, 1998). La concentrazione del settore della distribuzione al dettaglio e la sua posizione di controllo nell'ambito della catena di offerta agro-alimentare sono i presupposti che consentono di adottare, implementare e far rispettare standard privati (Fulponi, 2006). Conseguentemente, il rispetto degli standard privati da elemento di competitività si tramuta in semplice mezzo per restare sul mercato. E stato evidenziato come l'adesione a certi standard possa essere un prerequisito per l'accesso al mercato, poiché i costi aggiuntivi connessi con l'implementazione degli standard non sono sempre sufficientemente compensati dalla valorizzazione del prodotto (Henson, 2006).

\section{Aumento delle diseguaglianze ed esclusione}

Oggetto della contestazione è il modo in cui i costi e i benefici connessi all'adesione ai regimi di standardizzazione privati si ripercuotono lungo le catene di offerta e più in generale sul sistema agroalimentare. È stato osservato che per rispettare i protocolli richiesti dai dettaglianti, i produttori sono costretti a sostenere investimenti strutturali specifici, che si configurano come costi irrecuperabili e che possono essere da ostacolo per l'ingresso nel mercato, soprattutto per i piccoli produttori. Inoltre, l'efficienza generata dalle imprese dominanti, in particolare dalle grandi catene di distribuzione non sembra che si traduca in una equa distribuzione del valore creato, con effetti sfavorevoli, particolarmente con riferimento alla fase agricola (Sexton, 2010). L'impatto dei sistemi di governance privati sulla struttura e il modus operandi dei mercati mondiali dei prodotti agroalimentari sembra essere quello di un ulteriore consolidamento del potere delle imprese dominanti (Swinnen e Maertens, 2007).

\section{Barriere per il commercio internazionale}

I problemi di esclusione possono essere particolarmente gravi per le imprese dei paesi in via di sviluppo. Al fine di gestire i rischi associati alla globalizzazione delle fonti di approvvigionamento, a fronte delle crescenti preoccupazioni circa la sicurezza degli alimenti e ai requisiti di qualità richiesti dai consumatori, i buyers dominanti adottano norme private per gestire il rischio e/o differenziare i prodotti e per ridurre al minimo i costi di transazione ad essi associati, creando ostacoli ritenuti ingiustificati nel commercio internazionale, in particolare nei confronti dei paesi in via di sviluppo. Nonostante l'ampia letteratura a questo riguardo agiti lo spettro dell'esclusione (Korinek et al., 2008), le prove riguardo agli effetti degli standard privati sul commercio internazionale sono ben lungi dall'essere conclusivi, anche se si riconosce la necessità di promuovere programmi a favore degli agricoltori poveri e dei piccoli produttori con il compito di assisterli nel percorso di adeguamento (Maertens e Swinnen, 2008; Swinnen e Maertens, 2007).

\section{Legittimità e armonizzazione delle norme}

È messa in discussione la legittimità dei sistemi di governance privati, poiché i processi di formazione degli standard eludono i principi di trasparenza, rappresentatività e partecipazione, violando in tal modo il principio di equità (Henson e Reardon, 2005; Henson e Humphrey, 2009). Le critiche mosse s'incentrano sul fatto che i processi di standardizzazione privati si basano su relazioni top down ed autoritative, riguardo agli interessi degli attori che operano lungo le catene di offerta, con effetti distortivi che derivano dalle loro relazioni asimmetriche (squilibri nelle relazioni di potere). Altri aspetti critici riguardano la loro scarsa capacità di rappresentare esigenze più ampie e di comprendere gli interessi dei paesi in via di sviluppo, di non promuovere processi di armonizzazione, operando anzi in competizione con gli accor- di SPS nell'ambito del WTO e del Codex Alimentarius, entrando sostanzialmente in conflitto con le regole del commercio internazionale (Havinga, 2006). Infine, i processi di standardizzazione privati sono criticati perché seguirebbero un approccio non-scientifico, né sottoposto ad una verifica pubblica che ne provi la fondatezza quanto a livelli di protezione e/o di efficienza forniti (WTO, 2008).

\section{Considerazioni conclusive}

La sicurezza e la qualità rappresentano i principi funzionali su cui principalmente si sono andate sperimentando nuove forme di governance delle catene di offerta agroalimentare: l'inserimento di molteplici standard e l'imperfezione dei mercati condizionano le modalità organizzative degli scambi. La scelta della struttura di governance per gestirli è in funzione della strategia sviluppata dagli attori allo scopo di fronteggiare l'asimmetria informativa (Mènard, Valceschini, 2005; Raynaud et al. 2005). Pertanto soluzioni organizzative concertate $\mathrm{e}$ condivise di coordinamento potrebbero costituire in prospettiva i fattori chiave di strategie maggiormente adatte a competere nelle catene di offerta, dominate dalla grande distribuzione al dettaglio (Rama, 2010).

La vasta gamma di standard privati, il complesso intreccio di attori che intervengono negli assetti regolativi, la diversità di istituzioni cui questi hanno dato luogo, non consentono di trarre conclusioni generali quanto a opportunità e minacce dei sistemi privati di governance, in particolare riguardo ai suoi effetti sul commercio dei paesi in via di sviluppo (Henson e Humphrey, 2009). Tuttavia, con riferimento ai paesi in via di sviluppo e poveri, si riconosce l'opportunità di sviluppare un approccio policy oriented con particolare attenzione a politiche lungimiranti di adeguamento agli standard sanitari e fitosanitari internazionali ed una appropriata implemetazione che include, tra l'altro, maggiori risorse finanziarie e tecniche, infrastrutture per facilitare l'accesso alle tecnologie avanzate, strutture di informazione e formazione tecnica e gestionale (Maertens e Swinnen, 2008; Henson e Humphrey, 2009; Swinnen e Maertens, 2007; Shafaeddin, 2007).

Quanto ai processi di integrazione della regolazione pubblico-privata, molto probabilmente saranno i sistemi di governance privati a giocare in futuro il ruolo maggiore nel definire il riposizionamento e lo sviluppo della regolazione pubblica a livello nazionale e internazionale. Quest'ultima, però, continuerà a giocare un ruolo cruciale nel sostenere ed integrare le strategie private di sicurezza e qualità e favorire i processi di allineamento (Henson e Humphrey, 2009).

\section{Bibliografia}

Akerlof G.A., 1970. The market for lemons: Quality uncertainty and the market mechanism. Q. J. Econ. 84:488-500.

Bernstein S., Cashore B., 2007. Can non-state global governance be legitimate? An analytical framework. Regul. Gov. 1:347-371.

Codron J.M., Giraud Heraud E., Soler L.G., 2005. Minimum Quality Standards, Premium Private Labels, and European Meat and Fresh Produce Retailing. Food Policy 30:270-283.

Coff C., Korthals M., Barling D., 2008. Ethical traceability and informed food choice. In: C. Coff, D. Barling, M. Korthals and T. Nielson (eds.) Ethical Traceability and Communicating Food. Springer, London, UK.

de Stefano F., 2009. Problematiche economico-sociali nei Paesi avanzati sulla rintracciabilità e sulla sicurezza delle produzioni agroalimentari. pp 31-78 in Atti XLIV Convegno di Studi SIDEA Produzioni agroalimentari tra rintracciabilità e sicurezza. Analisi economiche e politiche d'intervento. Franco Angeli Ed., Milano, Italy. 
FA0, 2010. Draft report on a global survey on private standards, codes of conduct and guidelines in the livestock sector. Working document. FAO Publ., Roma, Italy.

Fulponi L., 2006. Private voluntary standards in the food system: The perspective of major food retailers in OECD countries. Food Policy 31:1-13.

Garcia Martinez M., Fearne A., Caswell J.A., Henson S.J., 2007. Co-regulation as a possible model for food safety governance: opportunities for public-private partnerships. Food Policy 32:299-314.

Giraud-Héraud, E., Grazia, C., Hammoudi, H., 2008. Regolazione della sicurezza sanitaria e comportamento strategico degli attori: una rassegna della letteratura. In: C. Grazia, R. Green e A. Hammoudi (eds.) Qualità e sicurezza degli alimenti. Una rivoluzione nel cuore del sistema agroalimentare. Franco Angeli Ed., Milano, Italy.

Giraud-Héraud E., Rouached L., Soler L.G., 2006. Private labels and public quality standards: How can consumer trust be restored after the mad cow crisis? QME-Quant. Mark. Econ. 4:31-55.

Green R., Perito M.A., 2008. Sicurezza alimentare e standard di qualità: verso una co-regulation tra il settore pubblico e privato. In: C. Grazia, R. Green e A. Hammoudi (eds.) Qualità e sicurezza degli alimenti. Una rivoluzione nel cuore del sistema agroalimentare. Franco Angeli Ed., Milano, Italy.

Grunert K.G., 2005. Food quality and safety: consumer perception and demand. Eur. Rev. Agric. Econ. 32:369-391.

Hammoudi A., Hoffmann R., Surry Y., 2009. Food safety standards and agri-food supply chains: An introductory overview. Eur. Rev. Agric. Econ. 36:469-478.

Havinga T., 2006. Private regulation of food safety by supermarkets. Law Policy 28:515-533.

Henson S., 2006. The role of public and private standards in regulating international food markets. Paper prepared for the IATRC Summer Symp. Food Regulation and Trade: Institutional Framework, Concepts of Analysis and Empirical Evidence, May 28-30, 2006, Bonn, Germany.

Henson S., Caswell J., 1999. Food safety regulation: an overview of contemporary issues. Food Policy 24:589-603.

Henson S.J., Humphrey J., 2009. The impacts of private food safety standards on the food chain and on public standard-setting processes. Paper Prepared for FAO/WHO, May 2009, ALINORM 09/32/9D Part II. Roma, Italy.

Henson S.J., Humphrey J., 2008. Understanding the Complexities of Private Standards in Global Agri-Food Chains. Grey Literature, Amsterdam, The Netherlands.

Henson S.J., Northen J.R., 1998. Economic determinants of food safety controls in the supply of retailer own-branded products in the UK. Agribusiness 14:113-126.

Henson S.J., Reardon T., 2005. Private Agri-Food Standards: implications for food policy and the agri-food system. Food Policy 30:241253.

Korinek J., Melatos M., Rau M., 2008. A review of methods for quantifying the trade effects of standards in the agri-food sector. OECD Trade Policy Working Papers No. 79. OECD Publ., Paris, France.

Lascoumes P., Le Galès P., 2009. Gli strumenti per governare. Mondadori Paravia Ed., Milano, Italy.

Liu P., 2009. Private standards in international trade: issues and opportunities. Presentation at the WTO/CTE workshop on private standards, July 2009. Roma, Italy.

Malorgio G., Grazia C., 2009. Strategie produttive e commerciali della sicurezza alimentare: lo standard GlobalGap e il ruolo delle Organizzazioni di Produttori. pp 253-261 in Atti XLIV Convegno di Studi SIDEA Produzioni agroalimentari tra rintracciabilità e sicu- rezza. Analisi economiche e politiche d'intervento. Franco Angeli Ed., Milano, Italy.

Maertens M., Swinnen J.F.M., 2008. Standards as barriers and catalysts for trade, growth and poverty reduction. J. Int. Agr. Trade Dev. 4:4762.

Ménard C., 2004. The economics of hybrid organizations. J. Inst. Theor. Econ. 160:345-376.

Ménard C., Valceschini, E., 2005. New institutions for governing the agri-food industry. Eur. Rev. Agr. Econ. 32:421-440.

OECD, 2007. Private standard schemes and developing country access to global value chains: challenges and opportunities emerging from four case studies. AGR/CA/APM. Publ., Paris, France.

Pulina P., 2010. Autorità, responsabilità e funzioni della distribuzione alimentare moderna. pp 39-68 in Atti XLVI Convegno di Studi SIDEA Cambiamenti nel sistema alimentare: Nuovi problemi, strategie, politiche. Franco Angeli Ed., Milano, Italy.

Rama D., 2010. Ruolo ed evoluzione delle istituzioni di marketing ("hybrids") nei nuovi scenari competitivi del sistema agro-alimentare. pp 95-130 in Atti XLVI Convegno di Studi SIDEA Cambiamenti nel sistema alimentare: Nuovi problemi, strategie, politiche. Franco Angeli Ed., Milano, Italy.

Raynaud E., Sauvée L., Valceschini E., 2005. Alignment between quality enforcement devices and governance structurrs in the agrofood vertical chains. J. Manage. Gov. 9:47-77.

Reardon T., Codron J.-M., Busch L., Bingen J., Harris C., 2001. Gobal change in agrifood grades and standards: agribusiness strategic responses in developing countries. Int. Food Agribusiness Manage. Rev. 2:421-435.

Sexton R.J., 2010. Forces shaping world food markets and the role of dominant food retailers. pp 71-94 in Atti XLVI Convegno di Studi SIDEA Cambiamenti nel sistema alimentare: Nuovi problemi, strategie, politiche. Franco Angeli Ed., Milano, Italy.

Shafaeddin M., 2007. Who does bear the costs of compliance with sanitary and phytosanitary measures in poor countries? MPRA Paper, University Library of Munich, Germany.

Smith G., 2009. Interaction of public and private standards in the food chain. OECD Food, Agriculture and Fisheries Working Papers No. 15. OECD Publ., Paris, France.

Swinnen J.F.M., Maertens M., 2007. Globalization, agri-food standards and development. Rivista di Economia Agraria a. LXII n. 3:413-421.

UNCTAD, 2007. Food safety and environmental requirements in export markets - friend or foe for producers of fruit and vegetables in asian developing countries? UN Publ., Geneve, Switzerland.

Williamson 0.E., 1996. The mechanisms of governance. Oxford University Press, Oford, UK.

World Bank, 2005. Food Safety and Agricultural Health Standards: Challenges and Opportunities for Developing Country Exports. World Bank, Washington, DC, USA.

WTO, 2007. Private standards and the SPS Agreement. WTO, Committee on Sanitary and Phytosanitary Measures, G/SPS/GEN/746. Available from: http://ec.europa.eu/food/international/organisations/sps/docs/g-sps-gen_746.pdf

WTO, 2008. Considerations Relevant to Private Standards in the Field of Animal Health, Food safety and Animal Welfare. WTO, Committee on Sanitary and Phytosanitary Measures. Available from: http://ec.europa.eu/food/international/organisations/sps/ docs/G-SPS-GEN-822.pdf

Zamagni S., 2006. Sicurezza degli alimenti, sviluppo sostenibile, qualità. In: G. Martino, C. Perugini e T. Sediari (eds.) La sicurezza degli alimenti. Contributi all'analisi economica. Donzelli Ed., Roma, Italy. 